\title{
A Transient Stability Assessment Method using Post-Fault Trajectories
}

\author{
Theodoros Kyriakidis, Guillaume Lanz, Rachid Cherkaoui, Maher Kayal \\ Electronics Laboratory \& Power System Group \\ Swiss Federal Institute of Technology \\ CH-1015 Lausanne, Switzerland \\ theodoros.kyriakidis@epfl.ch
}

\begin{abstract}
Transient Stability Assessment (TSA) is the process in which the stability of a system is characterized qualitatively or quantitatively. The TSA algorithm presented in this paper is derived from the well-established Single Machine Equivalent (SIME) method and can thus be categorized as a hybrid directtemporal method. The novelty of the proposed algorithm is that it derives a Transient Stability Index (TSI) with a single TimeDomain (TD) simulation for both stable and unstable cases. The resulting TSI is uniform in units and linear around the instability point. Results are reported for two sample power systems of 9 and 36 buses. The proposed algorithm has also been successfully employed to speed-up a Critical Clearing Time (CCT) determination algorithm.
\end{abstract}

Index Terms--power system dynamics, SIME method, timedomain simulation, transient stability assessment

\section{INTRODUCTION}

Transient stability is defined as the ability of the machines of a power system to retain their synchronism with the rest of the grid, after a given disturbance [1]. While transient stability is an inherent function of the power system, secure operation can be facilitated by the availability of adequate analytical tools [2]. One such tool is Transient Stability Assessment. It consists of the quantitative or qualitative characterization of the ability of the system to retain a state of operating equilibrium after being subjected to severe disturbances.

This paper presents a quantitative TSA algorithm that builds on the well-established SIME method [3]. Salient feature of the proposed algorithm is the ability to consistently derive a unit-uniform Transient Stability Index (TSI) with a single TD simulation for stable and unstable cases alike. Resulting TSIs exhibit linearity around the instability point and monotonicity against increasing fault duration time.

This paper is structured as follows. Section II introduces the reader to TSA principles. The proposed algorithm is presented in section III together with its mathematical foundation. In section IV the application of the algorithm on sample test systems is demonstrated. Finally, section $\mathrm{V}$ gives conclusions and suggests issues to be tackled in future work.

\section{TSA FUNDAMENTALS}

Depending on how the TSI is derived, TSA methods can be classified into time-domain simulation, direct, and hybrid.

Time-domain simulation methods perform the numerical integration of the differential-algebraic equations (DAE) governing the behavior of the power system. The main advantages include very accurate results guaranteed by welldeveloped Step-By-Step (SBSI) tools and the unlimited modeling capability of system elements. The most serious drawbacks are the computational burden for the solution of the complex DAEs and the fact that TD methods only provide a qualitative yes-or-no to the stability issue.

In direct methods, transient stability is investigated by forming an apposite Lyapunov transient energy function (TEF), which describes the energy in the power network. Stability is quantified by measuring the system's ability to "absorb" the surplus energy injected during the fault-on period so that the synchronous machines may reach a new postdisturbance equilibrium state. Drawbacks of direct methods include limited modeling capability, non-convergence issues especially in stressed situations [4], and the generation of rather conservative results.

Hybrid methods merge the above trying to mitigate disadvantages while retaining their virtues. They combine TD simulation with a TEF calculation of the degree of stability, to provide an index similar to the one of direct methods [4]-[7].

Hitherto, there are two key concerns regarding the majority of hybrid methods. First, they require functional integration into the TD program, which might not always be possible/practical. Second, derivation of the TSI is troublesome for stable cases. In most methods, additional pseudo-faults are applied as part of the process to derive the stable-case index, slowing down the procedure. In an effort to circumvent the above, the following requirements have been a priori specified for the proposed algorithm.

i. No modification or control in the TD engine should be available.

ii. Derive TSI with a single TD run for all cases.

iii. The TSI should be unit consistent over all its range.

iv. The resulting TSI should feature linearity especially around the critical point (stable to unstable transition). 


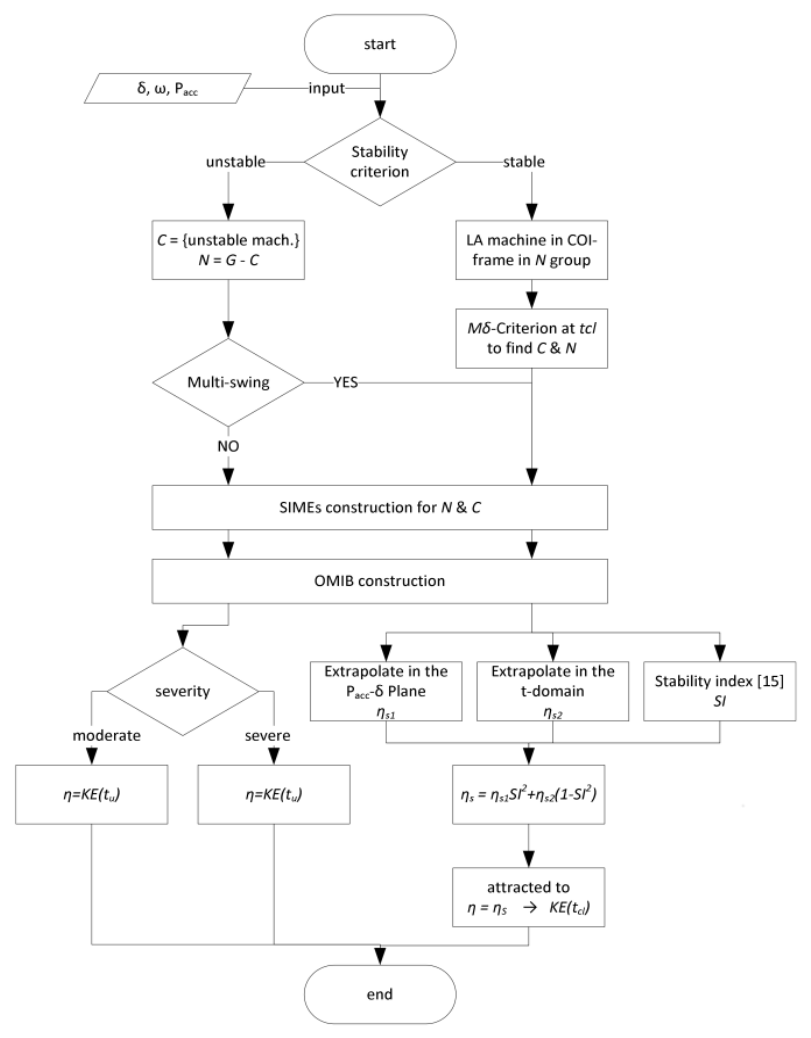

Figure 1 TSA algorithm overview

\section{PROPOSED ALGORITHM}

Fig. 1 presents an overview of the proposed algorithm. Its foundation is the well-established SIME concept [3]. Its output is a TSI that has units of transient energy. Stable cases result in positive TSI values and conversely unstable to negative ones. The more stable the case, the higher the TSI value, and vice versa. Functional blocks of the algorithm are explained in paragraphs that follow.

\section{A. Stability Criterion}

Classification of a case as stable or unstable is done according to a maximum angle separation criterion. If for any time, the angular separation between any two synchronous machines in the system exceeds a predefined threshold, then the case is deemed unstable [9]. In this work a threshold of $\delta_{t h}=180^{\circ}$ has been used.

\section{B. Unstable cases}

Mode of disturbance (MOD) determination is the separation of the machines of the system in two groups, critical $C$ and non-critical $N$ machines. When the case is unstable, then MOD determination is straightforward: machines that exhibit angular separation higher than $\delta_{t h}$ from the least advanced machine are deemed unstable.

In such cases, the accelerating power $P_{a}$ of the SIME turns negative after fault clearance, temporarily decelerating the system, before turning positive again, for the aperiodic oscillation. The system escapes the attraction of its Stable Equilibrium Point (SEP) when it starts accelerating again. That moment is termed the time to instability $t_{u}$, and the following holds $P_{a}\left(t_{u}\right)=0, \dot{P}_{a}\left(t_{u}\right)>0$. The TSI for unstable

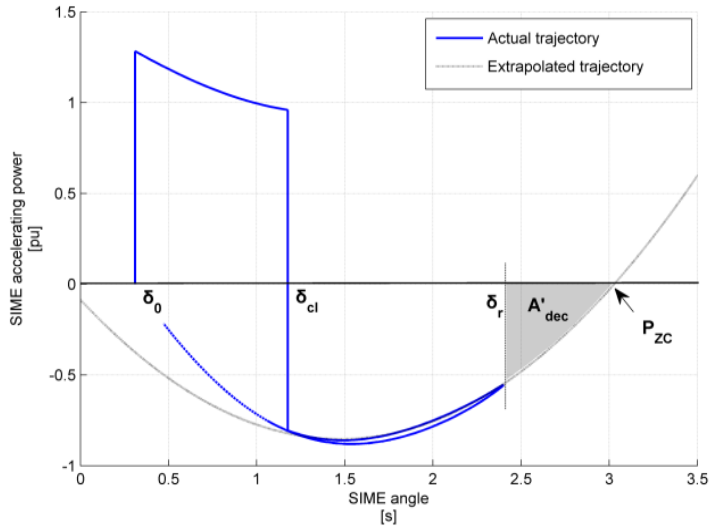

Figure 2 Extrapolation in the $\boldsymbol{P}_{\boldsymbol{a}}-\boldsymbol{\delta}$ for a stable case

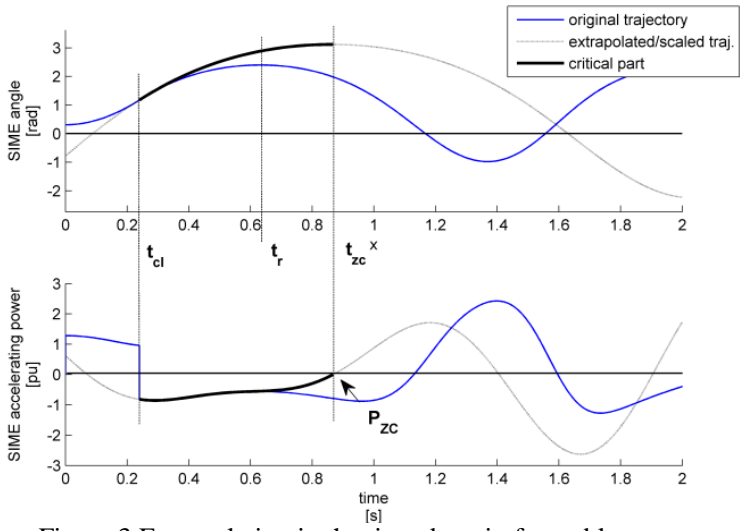

Figure 3 Extrapolation in the time domain for stable cases

cases is given by the excess kinetic energy of the SIME equivalent system at $t_{u}$.

$$
\eta=K E_{\text {corr }}\left(t_{u}\right)=-\frac{1}{2} M \omega\left(t_{u}\right)^{2}
$$

\section{Stable cases}

Stable cases are particularly challenging for two reasons. Firstly, MOD determination is non-trivial as all generators in the system retain their stability. Secondly, given a MOD, there is a time instant $t_{r}$ at which the angle of the resulting equivalent SIME reaches a maximum $\delta\left(t_{r}\right)=\delta_{r}$. The accelerating power remains negative until that moment. This means that if the system was to be driven unstable, there would be an unused extra decelerating period. In the $P_{a}-\delta$ plane, this period would appear as an extra decelerating area $A_{d e c}^{\prime}$, shaded in gray in fig. 2 . It is $A_{d e c}^{\prime}$ that accounts for the (positive) TSI.

1) $M O D$ determination: A maximum inertia-weighted angle gap criterion (called hereafter $M \delta$-criterion) applied at moment $t_{c l}$ has been used for MOD determination. Machines are sorted in descending order according to the product $M \delta_{i}=M_{i} \cdot \delta_{i}\left(t_{c l}\right)$. The maximum gap between sorted $M \delta_{i}$ values is identified, and machines before the gap are classified as $C$, whereas machines after the gap are classified as $N$. The $M \delta$-criterion can be seen as a normalized angular displacement of the machines due to the fault

2) Extra decelerating area approximation: In line with [3], this work proposes a TSI based on extrapolation of 


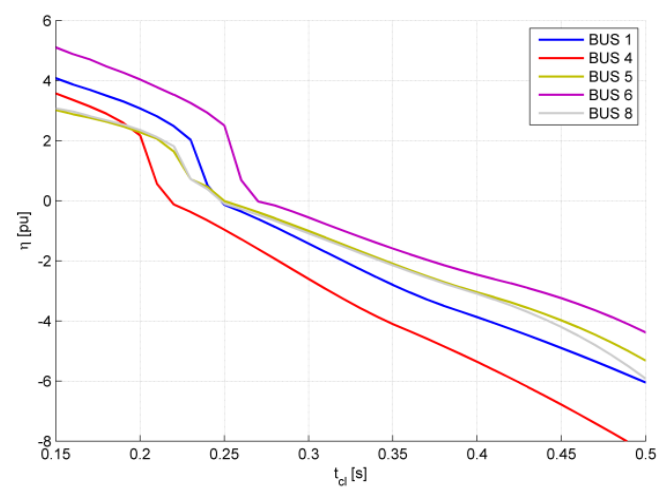

Figure 4 TSI results for the 9-bus system

trajectories, as a way of guessing the evolution of system dynamics. Extrapolation in different domains has been found to perform variedly under different circumstances. So, a composite index (combination of subindexes) is suggested.

Firstly, extrapolation is performed on the $P_{a}-\delta$ plane and the first subindex is calculated as $\eta_{S 1}=A_{d e c}^{\prime}$. Fig. 2 illustrates the concept. Accelerating power $P_{a}(\delta), \delta \in\left(\delta\left(t_{c l}\right), \delta_{r}\right]$ is approximated by $\hat{P}_{a}(\delta)$ using a $3^{\text {rd }}$-order polynomial.

A second subindex $\eta_{S 2}$ is derived using extrapolation in the time domain, namely on the $P_{a}-t$ and $\delta-t$ planes, as illustrated in fig. 3. $P_{a}(t)$ is approximated for $t>t_{c l}$ by $\hat{P}_{a}(t)$. Curve fitting is performed using a two-term sum-of-sines $\hat{P}_{a}(t)=a_{1} \sin \left(b_{1} t+c_{1}\right)+a_{2} \sin \left(b_{2} t+c_{2}\right)$. Because of the inherent periodicity of the sum-of-sines $\hat{P}_{a}(t)$, it is expected to have a zero-crossing at some time point $t_{z c}:\left.\hat{P}_{a}(t)\right|_{t=t_{z c}}$. Then, a scaling factor is defined as $s=\frac{t_{z c}-t_{c l}}{t_{r}-t_{c l}}$. This factor is used to scale $\delta(t) \stackrel{\times s}{\rightarrow} \hat{\delta}(\mathrm{t})$ so that the latter has a maximum at $t_{s c}$. Given $\hat{P}_{a}(t)$ and $\hat{\delta}(t)$, the SIME concept of unused decelerating area is employed to estimate the stability index as $\eta_{S 2}=A_{d e c}^{\prime}$.

Finally, akin to [8], the last subindex $S I \in[0,1]$ can be seen as a measure of how stable the case is.

$$
S I=\left(\frac{P_{a}\left(t_{r}\right)}{\min _{t \in\left(t_{c l} t_{r}\right)^{P_{a}(t)}}}\right)^{2}
$$

Once the three hereinabove described subindices have been calculated, they are combined as follows.

$$
\eta_{S}=\eta_{S 1} S I^{2}+\eta_{S 2}\left(1-S I^{2}\right)
$$

$S I^{2}$ has been used as a weight between subdindices $\eta_{S 1}$ and $\eta_{S 2}$, because it has been noted that $\eta_{S 2}$ provides a better estimate of the stability margin when the case is not strongly stable; vice versa for $\eta_{S 1}$. This can be explained by the fact that $\eta_{S 2}$ is based on a finer time-domain extrapolation, which is more precise the longer the disturbance has lasted. Whereas, $\eta_{S 1}$ is a roughly "blind" approximation on the $P_{a}-\delta$ plane, suitable when little information is available on the dynamics of the system.

All extrapolations have been done on a relative basis. This can sometimes lead to $\eta_{S}$ values unrealistically away from the

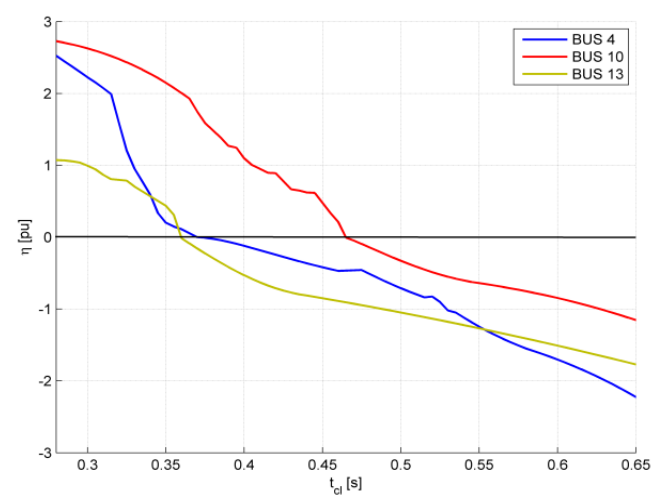

Figure 5 TSI results for the 36-bus system

scale of the system. A measure of the latter is the kinetic energy of the SIME equivalent at the time of fault clearance $K E_{c l}=-\frac{1}{2} M \omega^{2}\left(t_{c l}\right)$. With that in mind, an attractor function is used to attract the resulting $\eta_{S}$ towards more realistic values.

$$
f_{a t t}(x, c)=c+\operatorname{sign}(x-c)|x-c|^{1 / 2}
$$

Finally, the overall TSI is given by:

$$
\eta=f_{a t t}\left(\eta_{S}, K E_{c l}\right)
$$

Equation (5) has units of transient energy, and so, is unitconsistent with indices produced for unstable cases.

\section{NUMERICAL RESULTS}

The algorithm has been implemented in MATLAB, and applied on two sample power systems of 9-buses/3-generators and 36-bus/8-gen. All simulations were performed using the MatDyn time-domain simulator [10], in a typical modern PC.

\section{A. TSIs for a stable and an unstable case}

The event $A=\{$ Perfect $3-\varphi$ fault for $400 \mathrm{~ms}$ on bus \#10 $\}$ is considered for the 36-bus system. MOD identification takes place at the time of fault clearance $t_{c l}=0.4 \mathrm{~s}$ as described in section III-C1. Machines are sorted in descending $M \delta$ order, and the maximum gap is found between $\mathrm{G} 7$ and G4 $\left(M \delta_{\text {gap }}=\right.$ 4.517). So, the MOD is $N=\{G 1, G 2, G 3, G 4, G 5, G 6, G 8\}$ and $C=\{G 7\}$. Subindices $\eta_{S 1}, \eta_{S 2}$ and $S I$ are calculated as described in section III-C2, and the final TSI is given as follows.

$$
\begin{aligned}
\eta_{S 1} & =0.966 \\
\eta_{S 2} & =0.699 \stackrel{(3)}{\rightarrow} \eta_{S}=0.9044 \stackrel{(5)}{\rightarrow} \eta=1.097 \\
S I & =0.877
\end{aligned}
$$

Again for the 36-bus system, the event $B=\{$ Perfect 3- $\varphi$ fault for $600 \mathrm{~ms}$ on bus \#10\} is considered. Generator G7 loses its synchronism, so MOD identification is straightforward, $N=\{G 1, G 2, G 3, G 4, G 5, G 6, G 8\}$ and $C=\{G 7\}$. The TSI is calculated: (1) $\rightarrow-0.8468$

\section{B. TSI against fault duration}

Figures 4 and 5 show TSI results for different fault durations on sample buses of the systems. The horizontal axis of the graph corresponds to the sustained duration of the fault. Critical Clearing Time is defined as the duration above which a given fault would render the system unstable. In the graph 
this corresponds to the zero crossing of the respective TSI line. Naturally, cases turn unstable as the duration of the fault grows, that is, their TSI value crosses zero to the negative.

Monotonicity is maintained throughout the window of study. Linearity is high for the unstable part of the plot, and acceptable for the stable part. In most cases there is a sharp increase in the TSI for stable cases, as we move away from the critical point. This phenomenon is always system/casespecific, and can be attributed to the fact that less information on the dynamics of the system is available and as a result rougher approximations are made.

\section{Critical Clearing Time determination}

TSIs are commonly employed when the critical clearing time of a particular fault is investigated. When a TSI is not available CCT studies are usually performed in a "blind" binary search manner. Conversely, when it is available, information provided by the index can significantly speed up calculations, by reducing the number of required TD simulations. Examples of a "blind" and a TSI-enhanced CCT algorithm are presented in fig. 6. Particularities of the latter are highlighted in red.

Require: $\left[t_{\text {est }}^{l}, t_{\text {est }}^{u}\right]$ is the search window, and $t_{c}^{l}, t_{c}^{u}$ estimates for the bounds respectively; $e$ is the required precision and $N_{\text {max }}$ the maximum number of iterations allowed

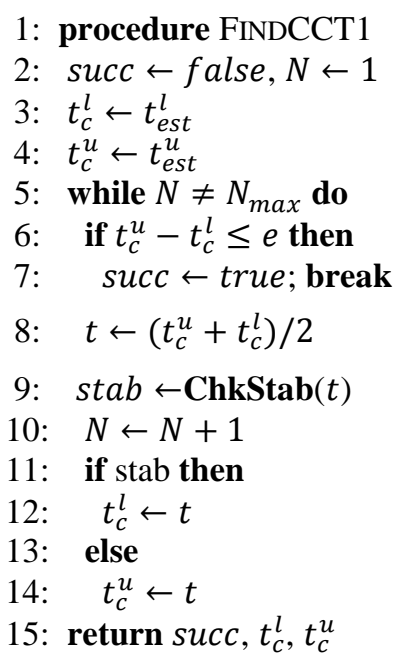

(a) "Blind" binary search

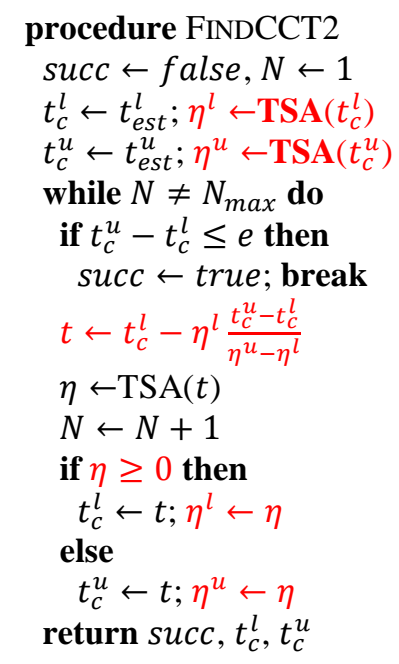

(b) TSI-based search
Figure 6 CCT determination algorithms

The algorithms of fig. 6 were tested on a $3 \varphi$ fault on bus \#10 for the 36-bus test system; similarly to events A and B of section IV-A. The search window was set to $\left[t_{\text {est }}^{l}, t_{\text {est }}^{u}\right]=$ $[0.200,1.000] s$ and a precision of $e=0.010 s$ was asked for. Table II summarizes the results of the iterations. Total speedup in time was $40.0 \%$. There was a $44.4 \%$ reduction in the number of TD simulations needed, $9 \rightarrow 5$. This percentage falls down to $36.4 \%$ if initialization is accounted for, $11 \rightarrow 7$.

\section{CONCLUSIONS}

This paper is dedicated to the assessment of transient stability of power systems. The importance of TSA applications was underlined and a SIME-based TSA algorithm was presented in detail, designed to meet specifically defined
TABLE I. RESULTS FOR A 3థ FAULT AT BUS \#10 OF THE 36-BUS SYSTEM

\begin{tabular}{ccc|ccc}
\hline & FINDCCT1 & \multicolumn{3}{|c}{ FINDCCT2 } \\
iter. \# & $t$ & stab & iter. \# & $t$ & $\eta$ \\
\hline $0_{1}$ & 0.2000 & yes & $0_{1}$ & 0.2000 & 2.978 \\
$0_{2}$ & 1.0000 & no & $0_{2}$ & 1.0000 & -3.606 \\
1 & 0.6000 & no & 1 & 0.5625 & -0.683 \\
2 & 0.4000 & yes & 2 & 0.4950 & -0.284 \\
3 & 0.5000 & no & 3 & 0.4700 & -0.478 \\
4 & 0.4500 & yes & 4 & $\mathbf{0 . 4 6 5 0}$ & -0.008 \\
5 & 0.4750 & no & 5 & $\mathbf{0 . 4 6 2 5}$ & +0.076 \\
6 & $\mathbf{0 . 4 6 2 5}$ & yes & & & \\
7 & 0.4700 & no & & & \\
8 & 0.4675 & no & & & \\
9 & $\mathbf{0 . 4 6 5 0}$ & no & & &
\end{tabular}

constraints. Results of the application of the algorithm on two different systems were presented, and the validity of the proposed method was demonstrated. Future work in the field is suggested to focus on improving the computational efficiency of the process, so that the proposed algorithm can be employed as a fast plug-and-play supplement to any existing TD simulation setup.

\section{ACKNOWLEDGMENTS}

This work is part of the Innovation Program on Energy of the EPFL Middle East.

\section{REFERENCES}

[1] IEEE/CIGRE Joint Task Force on Stability Terms and Definitions, "Definition and classification of power system stability," Power Systems, IEEE Transactions on, vol. 19, no. 3, pp. 1387-1401, Aug. 2004

[2] P. Kundur, G. Morison, and L. Wang, "Techniques for on-line transient stability assessment and control," in Power Engineering Society Winter Meeting, 2000. IEEE, vol. 1, Jan. 2000, pp. 46-51.

[3] M. Pavella, D. Ernst, and D. Ruiz-Vega, Transient stability of power systems: a unified approach to assessment and control, ser. Kluwer international series in engineering and computer science. Boston: Kluwer Academic Publishers, 2000.

[4] G. Maria, C. Tang, and J. Kim, "Hybrid transient stability analysis [power systems]," Power Systems, IEEE Transactions on, vol. 5, no. 2, pp. 384-393, May 1990.

[5] C. Tang, C. Graham, M. El-Kady, and R. Alden, "Transient stability index from conventional time domain simulation," Power Systems, IEEE Transactions on, vol. 9, no. 3, pp. 1524-1530, Aug. 1994.

[6] E. Vaahedi, Y. Mansour, and E. Tse, "A general purpose method for online dynamic security assessment," Power Systems, IEEE Transactions on, vol. 13, no. 1, pp. 243-249, Feb. 1998.

[7] D. Fang, A. David, C. Kai, and C. Yunli, "Improved hybrid approach to transient stability assessment", Generation, Transmission and Distribution, IEE Proceedings, vol. 152, no. 2, pp. 201-207, Mar. 2005.

[8] M. Haque, "Hybrid method of determining the transient stability margin of a power system," Generation, Transmission and Distribution, IEE Proceedings, vol 143, no. 1, pp. 27-32, Jan. 1996.

[9] R. Zarate-Minano, T. Van Cutsem, F. Milano, and A. J. Conejo, "Securing transient stability using time-domain simulations within an optimal power flow," IEEE Transactions on Power Systems, vol. 25, no. 1, pp. 243-253, 2010.

[10] S. Cole and R. Belmans, "MatDyn, a new MATLAB-based toolbox for power system dynamic simulation," Power Systems, IEEE Transactions on, vol. 26, no. 3, pp. 1129-1136, 2011. 